\title{
Magnetochromatic effects in magnetic fluid thin films
}

\author{
Herng-Er Horng, Chin-Yih Hong, Wai Bong Yeung, and Hong-Chang Yang
}

\begin{abstract}
A homogeneous ferrofluid composition capable of reversibly forming ordered crystalline twodimensional hexagonal lattices of magnetic particle columns in a thin film under the influence of external magnetic fields has been synthesized. We can manipulate the spacings between the particles columns by adjusting parameters such as external magnetic field strength, film thickness, rate of change of the field strength, and concentration of magnetic particles in the ferrofluid. These spacings between particle columns are of the order of several micrometers and are capable of diffracting visible light to produce monochromatic interference colors. We can change the resulting colors by altering the lattice spacing to exhibit the feasibility of generating monochromatic colors. (C) 1998 Optical Society of America

OCIS codes: $310.0310,210.3820,160.3820$.
\end{abstract}

\section{Introduction}

Ferrofluids are a type of magnetic fluid that typically consists of colloidal magnetic particles such as magnetite or manganese-zinc ferrites, dispersed with the aid of surfactants in a continuous carrier phase. The average diameter of the dispersed particles ranges between 5 and $10 \mathrm{~nm}$. Each particle has a constant magnetic dipole moment proportional to its size that can align with an external magnetic field.

Pattern-forming systems of magnetic fluid films under the influence of external magnetic fields have recently attracted much interest. To this end, a variety of different pattern-forming systems of magnetic fluid films have been studied, such as onedimensional patterns formed under the influence of fields oriented parallel to the plane of the field (parallel fields) $)^{1,2}$ and disordered two-dimensional lattices formed under the influence of fields oriented perpendicular to the plane of the film (perpendicular fields). ${ }^{3}$ Other investigators have generated

H.-E. Horng is with the Department of Physics, National Taiwan Normal University, Taipei 117, Taiwan. C.-Y. Hong is with the Department of Mechanical Engineering, Da-Yeh University, Chang-Hwa 515, Taiwan. W.-B. Yeung is with the Institute of Physics, Academia Sinica, Taipei 115, Taiwan. H.-C. Yang is with the Department of Physics, National Taiwan University, Taipei 106, Taiwan. Correspondence should be addressed to H.-E. Horng (e-mail: phyfv001@scc.ntnu.edu.tw).

Received 4 November 1997; revised manuscript received 7 January 1998.

0003-6935/98/132674-07\$15.00/0

(C) 1998 Optical Society of America more highly ordered two-dimensional lattices in thin films of magnetic fluid emulsions ${ }^{4}$ or magnetic fluids containing nonmagnetic spheres by using perpendicular magnetic fields. ${ }^{5}$ However, these lattices tend to solidify and therefore are not suitable for applications requiring rapid interconversion between crystalline and amorphous states.

Recently, we reported the synthesis of a homogeneous ferrofluid composition capable of reversibly forming well-ordered two-dimensional hexagonal lattices of magnetic particle columns in a thin film under the influence of external perpendicular fields. ${ }^{6}$ In this paper, we demonstrate how the lattice spacing between the particle columns can be manipulated by adjusting parameters such as the external magnetic field strength, the film thickness, the rate of change of the field strength, and the concentration of magnetic particles in the ferrofluid. The spacings are of the order of several micrometers and are therefore capable of diffracting visible light to produce monochromatic interference colors as white light passes through the lattice. One can change the resulting colors by altering the lattice spacing. This research demonstrates the feasibility of using homogeneous ferrofluids as new materials from which liquidcrystal-like magneto-optical devices may be developed.

\section{Homogeneous Magnetic Fluids}

The chemical synthesis of magnetite $\left(\mathrm{Fe}_{3} \mathrm{O}_{4}\right)$ by coprecipitation of $\mathrm{FeSO}_{4}$ and $\mathrm{FeCl}_{3}$ in the presence of $\mathrm{NaOH}$ is based on a reaction proposed by W.C. Elmore in 1938. ${ }^{7}$ This co-precipitation reaction has been used to generate ferrofluids in which the mag- 


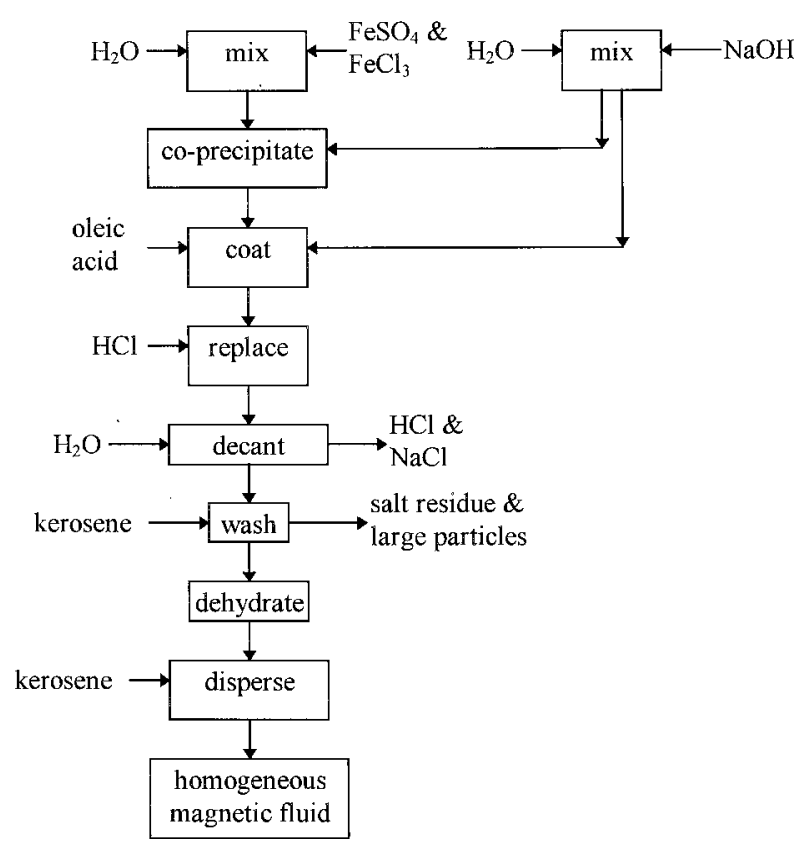

Fig. 1. Flow chart of the steps for preparation of a homogeneous ferrofluid capable of forming ordered two-dimensional lattices when a thin film of the fluid is subjected to an external magnetic field. An $8 \mathrm{M} \mathrm{NaOH}$ solution was added to the mixture of $\mathrm{FeSO}_{4} \cdot 7 \mathrm{H}_{2} \mathrm{O}$ and $\mathrm{FeCl}_{3} \cdot 6 \mathrm{H}_{2} \mathrm{O}$ to maintain the $\mathrm{pH}$ at 11.5 during the heating process. The co-precipitation of $\mathrm{Fe}_{3} \mathrm{O}_{4}$ occurs over an $\sim 20$-min time period. Oleic acid is added to the solution out of which the $\mathrm{Fe}_{3} \mathrm{O}_{4}$ has precipitated to coat the $\mathrm{Fe}_{3} \mathrm{O}_{4}$ particles and prevent agglomeration. This process takes $\sim 30 \mathrm{~min}$ and is followed by adding $\mathrm{HCl}$ to the reaction mix to protonate the carboxylate group on the oleic acid and thereby replace the $\mathrm{Na}^{+}$counter ion with a proton. The next step is decantation. During this step, deionized water is added to remove remaining counter ions of $\mathrm{HCl}$ and $\mathrm{NaCl}$ from the surfactant-coated $\mathrm{Fe}_{3} \mathrm{O}_{4}$ product. Washing is achieved by dispersing the settled $\mathrm{Fe}_{3} \mathrm{O}_{4}$ in kerosene. The mix is subjected to a short, low-speed spin in a centrifuge to remove remaining salt residues and large particles. The dehydration can be achieved by suspending the remaining $\mathrm{Fe}_{3} \mathrm{O}_{4}$ in acetone and drying it in a oven. After the particles have been dehydrated, they are again dispersed in kerosene, and the fluid is subjected to another short, low-speed spin in a centrifuge. This spin pellets larger or aggregated particles. The liquid sitting above any pellet that may be formed in this spin is the homogeneous magnetic fluid.

netite particles are coated with oleic acid (surfactant) and dispersed in kerosene (carrier). Figure 1 shows a flow chart of the steps used to prepare the homogeneous magnetic fluid used in the present research.

Based on the procedure outlined above, a homogeneous magnetic fluid is prepared. The x-ray diffraction patterns of the sample verified the singlephase fcc spinel structure expected for an $\mathrm{Fe}_{3} \mathrm{O}_{4}$ sample. The lattice constant is $8.40 \AA$. The magnetization of the sample is measured with a vibration sample magnetometer. We determined the particle size of the sample from the magnetization, applied field data ( $M-H$ data) by fitting it to the Langevin function $L(\alpha)=M / M_{s}=(\operatorname{coth} \alpha-1 / \alpha)$, where $\alpha=M_{s} V H / k T, M$ is the magnetization of the sample at an applied magnetic-field strength $H, M_{s}$ is the saturated magnetization of the sample, and $V$ is the volume of a particle. The saturated magnetization of the sample is $10.58 \mathrm{emu} / \mathrm{g}$, and the particle size is around $100 \AA$. The volume fraction of the homogeneous magnetic fluid was calculated as the ratio of the saturated magnetization of the magnetic fluid to that of the dry $\mathrm{Fe}_{3} \mathrm{O}_{4}$ powder. This ratio is $18.9 \%$.

\section{Two-Dimensional Lattices in Magnetic Fluid Thin Film}

Ferrofluid synthesized according to the procedure outlined above was sealed into a number of glass cells with various cell thicknesses to form fluidic thin films. These thin films were subjected to externally applied perpendicular magnetic fields generated by a pair of uniform solenoids. The applied fields were uniform; deviations of the field strength in the region of the film were measured with a gaussmeter and were found to be less than $1 \%$.

When a homogeneous magnetic fluid thin film is subjected to a perpendicular magnetic field, initial disorder quantum columns form. At a critical field strength $H_{l}$, an equilibrium two-dimensional hexagonal structure forms with particle columns occupying lattice vertices. If the field strength is increased to another critical value $H_{l}$, the pattern changes from a hexagonal structure to a labyrinthine pattern. Figure 2 illustrates this phenomenon in a thin film of thickness, $L=6 \mu \mathrm{m}$. In the range of field strengths between $H_{h}$ and $H_{l}$, the distance between the particle columns is roughly proportional to the inverse of the field strength.

We examined the effect of film thickness on pattern formation by varying film thickness from 10 to $2 \mu \mathrm{m}$ by using glass sample cells having different cell depths, while holding all other parameters constant. In Fig. 3 we provide examples of images of the thin film of the homogeneous magnetic fluid, using a constant field strength of 100 Oe. These images indicate that the distance between the particle columns is roughly proportional to the thickness of the film. In Fig. 4 we plot the distance between particle columns as a function of magnetic field strength and film thickness.

\section{Magnetochromatic Effects}

Diffraction phenomena occur as a parallel ray of white light passes through the two-dimensional column arrays formed in the homogeneous magnetic fluid thin film. Constructive and destructive interference occurs as the light rays reach the eyes of a viewer. The setup illustrated in Fig. 5 was used to demonstrate the magnetic chromatic effects resulting from the diffraction of light through the arrays. The area of the thin film used was $1 \mathrm{~cm} \times 4 \mathrm{~cm}$. Photographic images of the light through the film were taken with a conventional film camera.

Figure 6 is a photograph of a drop of a homogeneous magnetic fluid exposed to a perpendicular field. The thickness of the drop varies because of the surface tension of the fluid. Because the spacing of the 

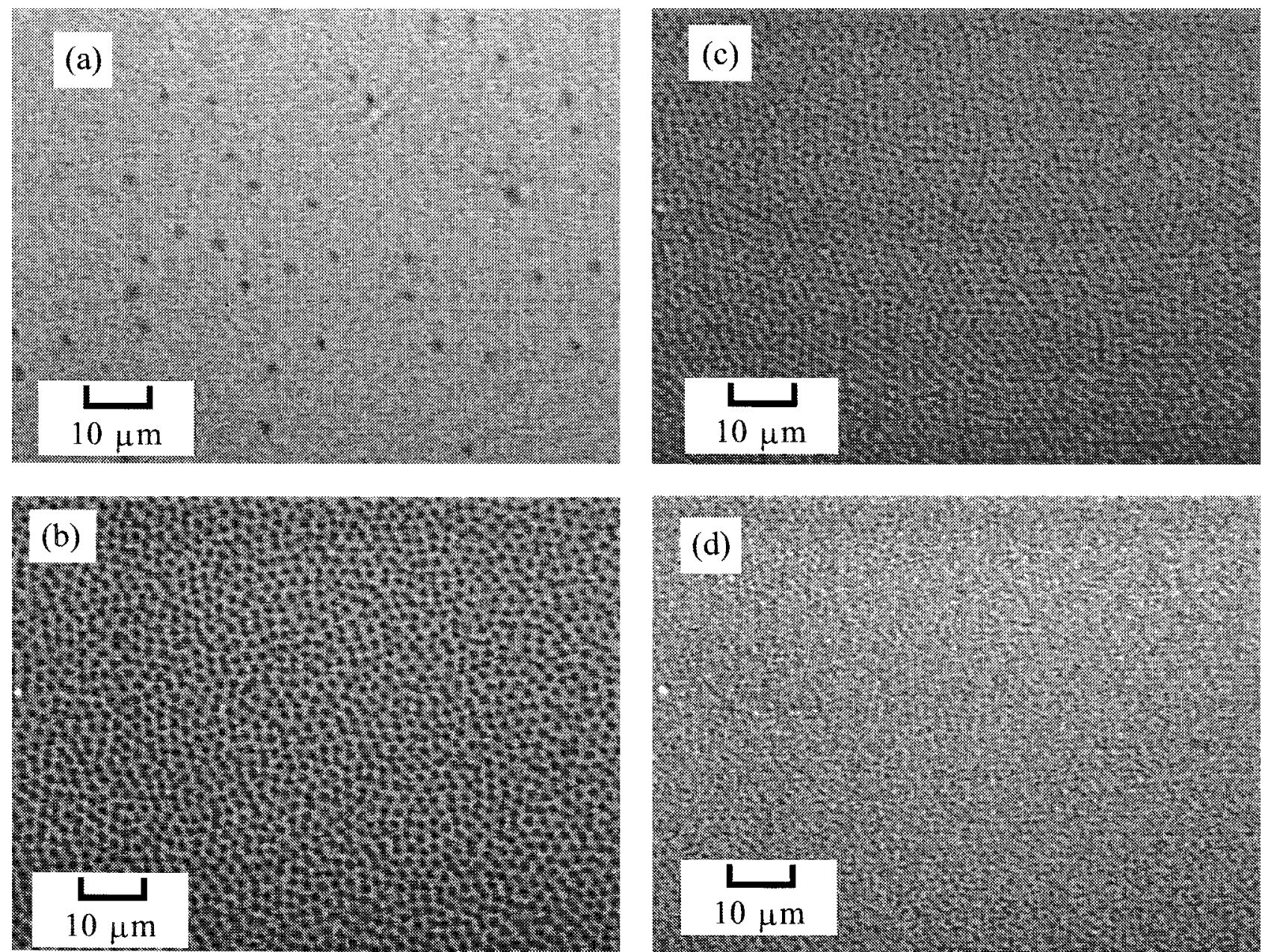

Fig. 2. Evolution of the pattern formation in a homogeneous ferrofluidic thin film in response to an externally applied magnetic field oriented perpendicular to the plane of the thin film. Images of the thin film under the influence of perpendicular magnetic fields were captured from a CCD video camera mounted on a Zeiss optical microscope, and written to computer. A simple computer program was written to control the image acquisition and the power to the solenoids used for generating the magnetic fields. This permitted easy manipulation of final field strength and its rate of change $(\mathrm{d} H / \mathrm{d} t)$. The scale bar on the image equals $10 \mu \mathrm{m}$ : (a) disorder quantum column, $H=70$ Oe, $L=6 \mu \mathrm{m}, \mathrm{d} H / \mathrm{d} t=20 \mathrm{Oe} / \mathrm{s}$; (b) hexagonal pattern, $H=100 \mathrm{Oe}, L=6 \mu \mathrm{m}, \mathrm{d} H / \mathrm{d} t=20$ Oe/s; (c) hexagonal pattern, $H=300 \mathrm{Oe}, L=6 \mu \mathrm{m}, \mathrm{d} H / \mathrm{d} t=20 \mathrm{Oe} / \mathrm{s}$; and (d) labyrinthine pattern, $H=400$ Oe, $L=6 \mu \mathrm{m}, \mathrm{d} H / \mathrm{d} t=20 \mathrm{Oe} / \mathrm{s}$.

ordered arrays formed in response to the field varies as a function of film thickness, a spectrum of colors is seen when a source of parallel white light is placed below the film.

Figure 7 is a series of photographic images that illustrate the diffraction of light by film as the field strength is varied. The color of the film changes from red to violet as the magnetic field is altered. These images demonstrate that the color of light passing through the thin film can be controlled and that monochromatic light can be obtained from a thin film with an area of the order of several square centimeters.

\section{Discussion}

Figure 8 is a cross-section drawing of arrays formed in a homogeneous magnetic fluid thin film illustrating the diffraction effects. In this figure $d$ is the distance between columns in the array, $\theta$ is the angle formed between the incoming light ray and the direc- tion perpendicular to the plane of the film, $\theta^{\prime}$ is the angle formed between the diffracted rays and the direction perpendicular to the plane of the film, and $N$ is the total number of magnetic particle columns diffracting the light. After diffraction, the intensity of the light $I$ is

$$
I \propto \frac{\sin ^{2}[(N \varphi) / 2]}{\sin ^{2}(\varphi / 2)},
$$

where $\varphi=[(2 \pi) / \lambda] d\left(\sin \theta+\sin \theta^{\prime}\right)$ and $\lambda$ is the wavelength of light. The condition in which the light intensity $I$ becomes maximum is the same as that in which the light becomes brightest after diffraction through the film. The condition is

$$
\frac{\varphi}{2} \equiv \frac{\pi}{\lambda} d\left(\sin \theta+\sin \theta^{\prime}\right)=\kappa \pi,
$$

where $\kappa$ is a nonnegative integer. 

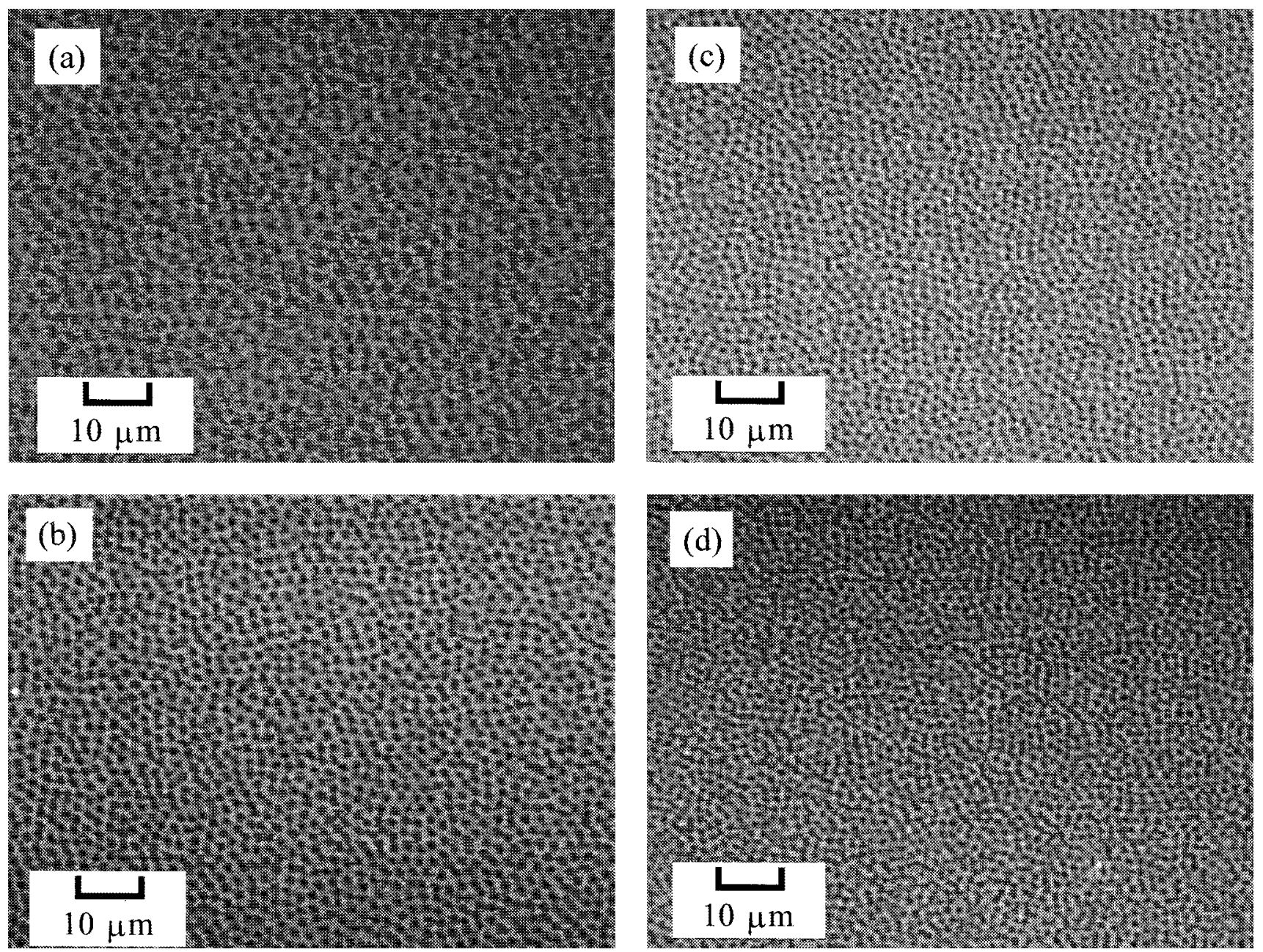

Fig. 3. Two-dimensional hexagonal arrays formed in films with different thicknesses in response to a perpendicular, 100-Oe magnetic field: (a) $L=10 \mu \mathrm{m}$, (b) $L=6 \mu \mathrm{m}$, (c) $L=4 \mu \mathrm{m}$, and (d) $L=2 \mu \mathrm{m}$.

If both $\theta$ and $\theta^{\prime}$ are not both equal to zero, the condition of $\kappa=0$ will never occur. The condition of $\kappa=1$ is the most interesting and important one. In this condition $d$ will be related to $\lambda$ by $d\left(\sin \theta+\sin \theta^{\prime}\right)$ $=\lambda$. If this wavelength $\lambda$ is within the range of visible light, the same $d$ will also allow only light with a wavelength of $\lambda / \kappa$ for $\kappa=2,3, \ldots$, to pass through the film. Fortunately, light with these wavelengths is outside the visible spectrum. The reason for this is that the longest wavelength of light visible to the human eye is $\sim 0.7 \mu \mathrm{m}$, and so the wavelength of $\lambda / 2=0.35 \mu \mathrm{m}$. This wavelength is the ultraviolet region of the electromagnetic spectrum and therefore is not visible to the human eye. Consequently, the viewer will observe only a single wavelength of light.

There will be dispersion for the intensity $I$. The degree of the dispersion $\delta \lambda$ must satisfy the condition $(\delta \lambda) / \lambda=1 / N$. In the case of a two-dimensional column array of a homogenous magnetic fluid thin film, $N$ is very large and depends on the area of the film. Thus $\delta \lambda / \lambda$ is very small. If the distance between columns $d$ satisfies $d=\lambda /\left(\sin \theta+\sin \theta^{\prime}\right)$, a pure monochromatic color will be observed. The distance
Distance between columns vs thickness

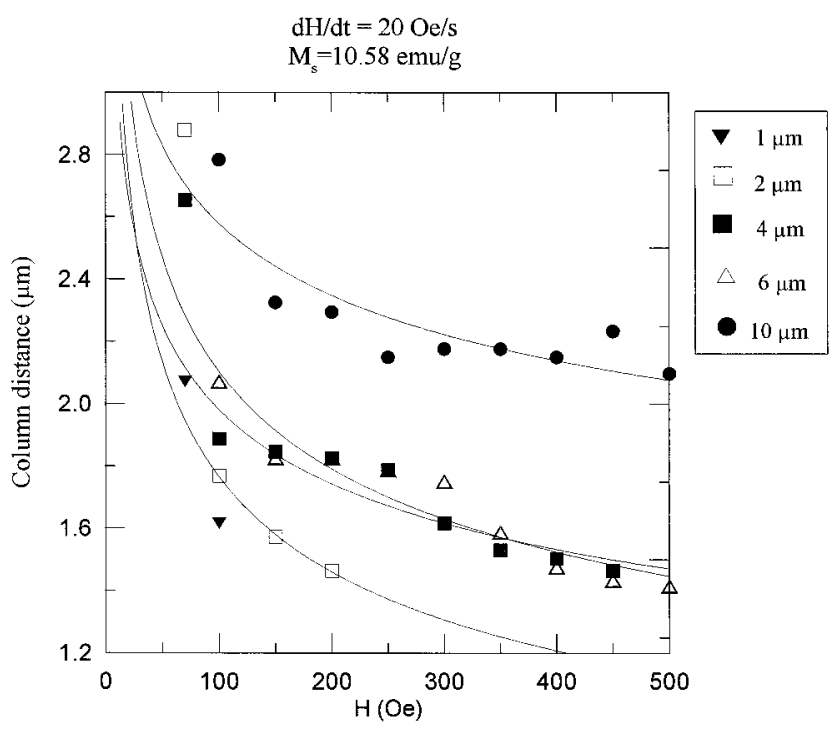

Fig. 4. Relation of the distance between particle columns in twodimensional hexagonal arrays to magnetic field strength and film thickness. 


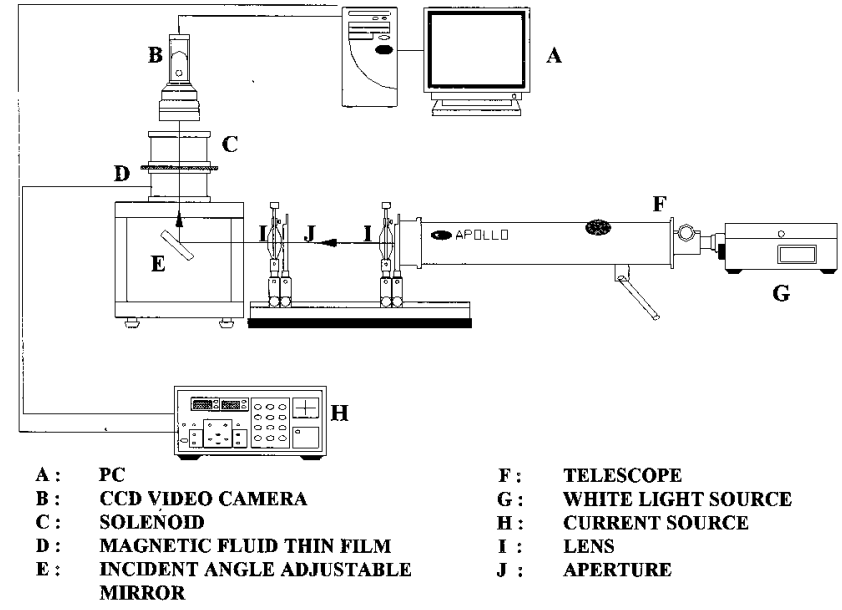

Fig. 5. Setup used for demonstrating light diffraction phenomena generated by ordered structures in homogeneous ferrofluidic thin film. A pair of uniform solenoids was used to generate perpendicular magnetic fields, and a halogen lamp was used to generate white light. The light rays were made to be near parallel by passing them through a telescope. Two optical lenses were used to make the near-parallel light parallel. An aperture was placed between the two lenses to control the size of the light beam. The parallel white light was reflected by a mirror located beneath the thin film. The angle of the mirror to the light beam was adjustable by turning the mirror plane, resulting in a change of the incident angle of the light to the film.

between columns in two-dimensional column arrays of homogeneous magnetic fluids is of the order of several micrometers. Therefore the array is capable of diffracting visible light to produce intensity interference. Furthermore, because distance $d$ can be manipulated by, e.g., controlling the strength of the externally applied magnetic field, the thickness of the magnetic fluid film and/or the angle $\theta$, the color of the light observed by the viewer can be changed at will.

This research lays the groundwork for future development of liquid-crystal-like displays by using homogeneous magnetic fluid thin films. One such device under development in our laboratory has pixel elements constructed with these magnetic fluid thin

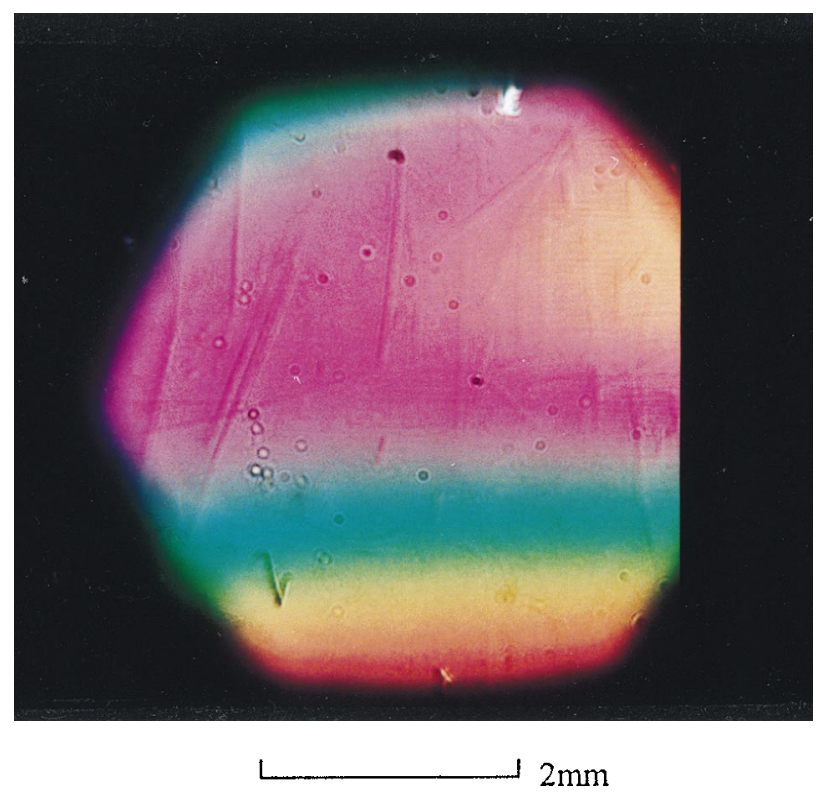

Fig. 6. Image of a spectrum of colors produced by a ferrofluidic drop in which the thickness of the drop varies from 2 to $10 \mu \mathrm{m}, H=$ $150 \mathrm{Oe}, \mathrm{d} H / \mathrm{d} t=500 \mathrm{Oe} / \mathrm{s}$. The scale bar corresponds to $2 \mathrm{~mm}$.

films with an independent electronic circuit for controlling the magnetic field. By properly adjusting the current in each pixel, we may display a polychromatic image.

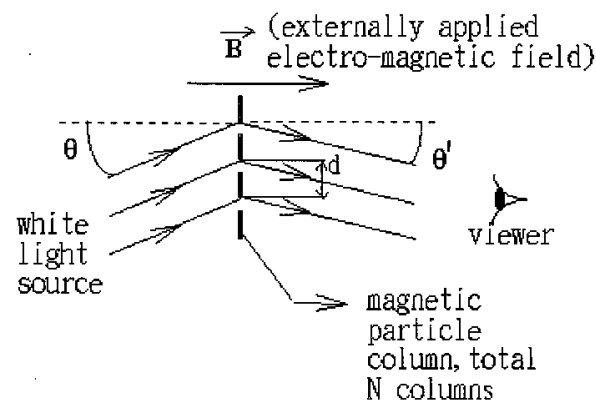

Fig. 8. Cross section of a homogeneous ferrofluidic thin film for the light diffraction concept. 

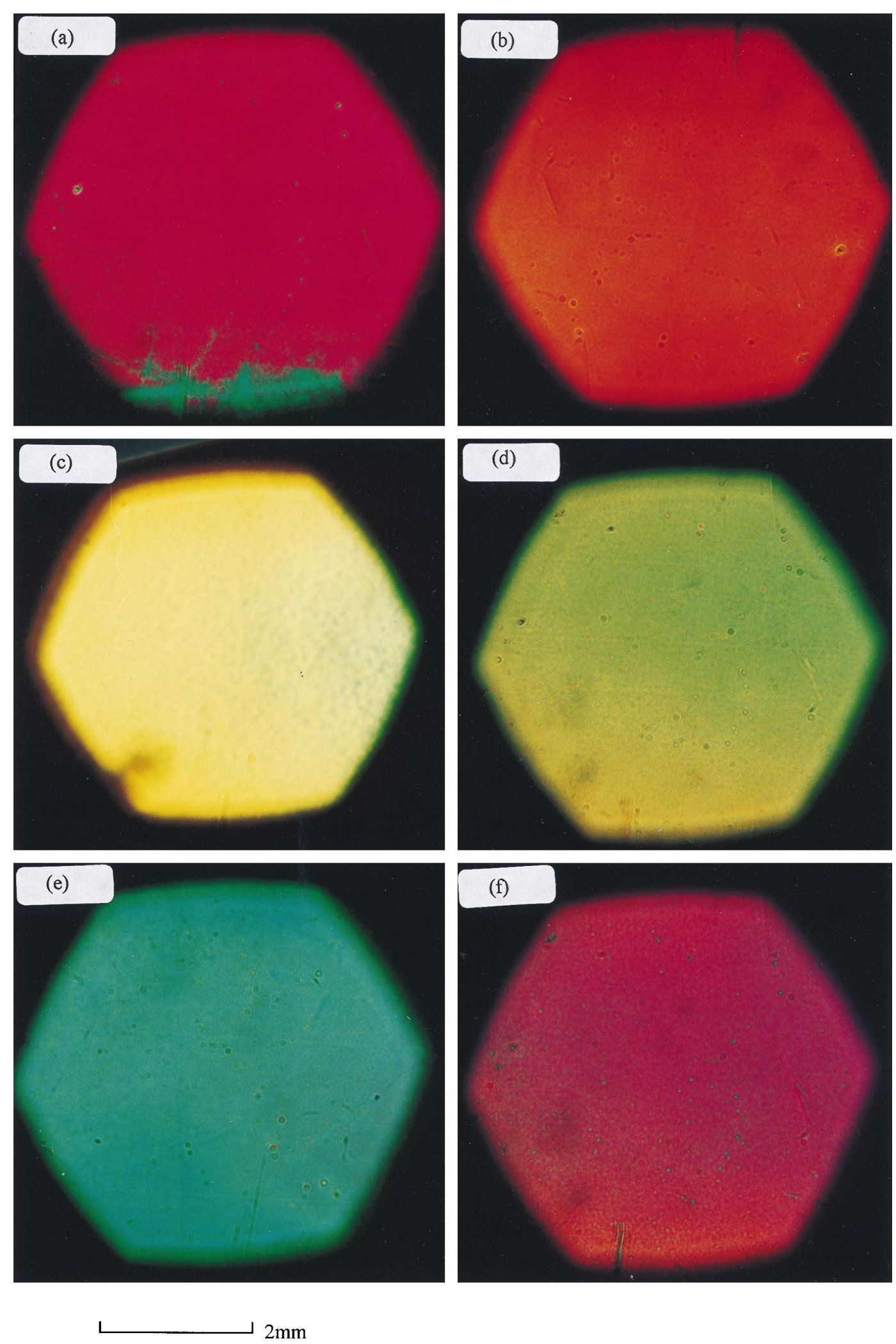

Fig. 7. Images showing different colors produced by a homogeneous ferrofluid thin film as the externally applied magnetic field strength varied. The scale bar corresponds to $2 \mathrm{~mm}$. 


\section{References}

1. M. Fermigier and A. P. Gast, "Structure evolution in a paramagnetic latex suspension," J. Colloid Interface Sci. 154, 522539 (1992).

2. D. Wirtz and M. Fermigier, "One-dimensional patterns and wavelength selection in magnetic fluids," Phys. Rev. Lett. 72, 2294-2297 (1994).

3. H. Wang, Y. Zhu, C. Boyd, W. Luo, A. Cebers, and R. E. Rosensweig, "Periodic branched structures in a phase-separated mag- netic colloid," Phys. Rev. Lett. 72, 1929-1932 (1994).

4. J. Liu, E. M. Lawrence, A. Wu, M. L. Ivey, G. A. Flores, K. Javier, J. Bibette, and J. Richard, "Field-induced structures in ferrofluid emulsions," Phys. Rev. Lett. 74, 2828-2831 (1995).

5. A. T. Skjeltorp, "One- and two-dimensional crystallization of magnetic holes," Phys. Rev. Lett. 51, 2306-2309 (1983).

6. C.-Y. Hong, I. J. Jang, H. E. Horng, C. J. Hsu, Y. D. Yao, and H. C. Yang, "Ordered structures in $\mathrm{Fe}_{3} \mathrm{O}_{4}$ kerosene-based ferrofluids," J. Appl. Phys. 81, 4275-4277 (1997).

7. W. C. Elmore, "Ferromagnetic colloid for studying magnetic structure," Phys. Rev. 54, 309-310 (1938). 